\title{
Mechanism of fat-binding and fat-contenting of the nanoparticles of the "Magnetofood" food additive
}

\author{
Iryna Tsykhanovska ${ }^{1}$, Victoria Evlash ${ }^{2}$, Oleksandr Oleksandrov ${ }^{1}$, \\ Tetiana Hontar ${ }^{1}$, Azret Shingisov ${ }^{3}$ \\ 1 - Ukrainian Engineering-Pedagogics Academy, Kharkiv, Ukraine \\ 2 - Kharkiv State University of Food Technology and Trade, Kharkiv, Ukraine \\ 3 - South Kazakhstan State University, M. Auezov, Shimkent, Kazakhstan
}

\begin{abstract}
The goal of research is the mechanism study of interaction of $\mathrm{Fe}_{3} \mathrm{O}_{4}$ nanoparticles ("Magnetofood" food additive) with linoleic acid and sunflower oil which is represented by the "Two-layer coordination" model. The chemical composition of the surface layers and the chemical state of elements on the surface of $\mathrm{Fe}_{3} \mathrm{O}_{4}$ nanoparticles coated with linoleic acid and unrefined sunflower oil was studied by X-ray photoelectron pectroscopy. It was established that on the spectra lack the $\mathrm{C} 1 \mathrm{~s}$ absorption band at $290 \mathrm{eV}$ which corresponds to carboxyl carbon $(-\mathrm{COOH})$, that is, there is no Estern group. Thisallows for the conclusionabout the fat-binding and fatcontentingcapacity of the nanoparticles of "Magnetofood" food additive.
\end{abstract}

Corresponding author: Iryna Tsykhanovska

E-mail: cikhanovskaja@gmail.com

DOI: $10.24263 /$ RES-2018-10

\section{Introduction}

One of the important functionally and technological properties of the food raw materials and the food ingredients which determines the technological processes course and thefinished product quality is the fat-retainingcapacity (FRC). FRCis the capacity to bind and retain fat. Fats are triglycerides. The edible fatspeciation. besides triglycerides, also contains the higher fatty acids (oleinic linoleic, linoleic ones are in oils; palmitic, stearic are in animal fats). Therefore, when studying of the FRC mechanism it is necessary to consider the physical chemical interactions of the food systems ingredients with the main components of fats and oils - triglycerides and higher fatty acids. Knowledge of the binding mechanisms and the fat content of the raw materials will allow the rational use of new types of the food raw materials and the food additives and predict the raw ingredients behavior in the food systems (dough and confectionery masses, minced meat, etc.) in the process of the processing and storage of finished products. 
Thus, the food fibers have the high fat-binding capability (FBC) of the mechanism of which is not well understood. There is an opinion that FRC and FBC are determined by lignin presence and are not affected by the particles size [1,2]. The following scientific studies of the mechanism of FRC and FBC and the food fibers in the meat systems [3-6] showed the dependence of the fat-retaining capacities and the fat burning properties on the number and size of the raw particles. This suggested that the fat absorption mechanism by the food fibers can be determined not only by the lignin sorption activity the availability of which increases during grinding, but also by the surface adsorption [3-6].

\section{Materials and methods}

\section{Research subjects}

Sample $1-\mathrm{Fe}_{3} \mathrm{O}_{4}$ nanoparticles coveredby linoleicacid. It was obtained by the dispergating $1 \mathrm{~g}$ of $\mathrm{Fe}_{3} \mathrm{O}_{4}$ nanoparticles (sample 1) and $0,2 \mathrm{~g}$ oflinoleic acid in $10 \mathrm{ml}$ of dymethylformamide for 12 hours at a temperature of $(50 \pm 1){ }^{\circ} \mathrm{C}$ and nitrogen stream blowing over the surface of the reaction mixture. After cooling the suspension to (2025) ${ }^{\circ} \mathrm{C}, \mathrm{Fe}_{3} \mathrm{O}_{4}$ nanoparticles coveredby linoleic acid were isolated by magnetic filtration and washed with the water-ethanol mixtures (1:1) in 5-7 times. The final product was dried in vacuum at $(60 \pm 1)^{\circ} \mathrm{C}$ for 24 hours;

Sample $2-\mathrm{Fe}_{3} \mathrm{O}_{4}$ nanoparticles coveredbythe unrefined sunflower oil or 1linoleyl-2-oleoyl-3-linole-noylglycerol. Dispersed $1 \mathrm{~g}$ of $\mathrm{Fe}_{3} \mathrm{O}_{4}$ nanoparticles (sample 1) and $0,2 \mathrm{~g}$ of the unrefined sunflower oil or 1-lino-leyl-2-oleoyl-3-linolenoylglycerol in $10 \mathrm{ml}$ of dimethylformamide for 12 hours at a temperature of $(50 \pm 1){ }^{\circ} \mathrm{C}$ and the nitrogen stream of above the surface of the reaction mixture. After cooling the suspension to (20-25) ${ }^{\circ} \mathrm{C}, \mathrm{Fe}_{3} \mathrm{O}_{4}$ nanoparticles covered bythe unrefined sunflower oil or 1-linoleyl-2-oleoyl-3-linolenoylglycerol were isolated by magnetic filtration and washed with the water-ethanol mixture (1:1) in 5-7 times. The final product was dried in vacuum at $(60 \pm 1){ }^{\circ} \mathrm{C}$ for 24 hours.

\section{X-ray photoelectron spectroscopy (XPES or XPS)}

The study of the chemical composition of the surface layers and the chemical state of the elements on the surface of the experimental samples 1, 2 was carried out on the Kratos Axis Ultra DLD electron spectrometer ("Kratos Analytical Limited", UK). As an exciting X-ray, the $\mathrm{Al} \mathrm{K}_{\alpha}$ line with photon energy hv $=1486,6 \mathrm{eV}$, voltage at the 15 $\mathrm{kV}$ tube and the emission current of $10 \mathrm{~mA}$ were used. The recording of XPE-spectra was carried out in the constant energy mode of the analyzer passing through which was $160 \mathrm{eV}$ when recording observation spectra and $40 \mathrm{eV}$ - for the registration of the spectra of the internal electronic levels of the main elements: Fe 2p, O 1s, C 1s. The scales of the association energy $\left(\mathrm{E}_{\mathrm{as}}\right)$ of the spectrometer were pre-calibrated on the 


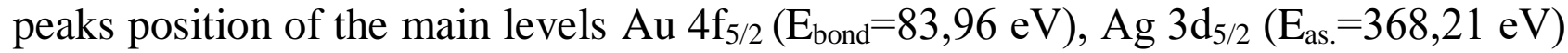
i $\mathrm{Cu} 2 \mathrm{p}_{3 / 2}\left(\mathrm{E}_{\mathrm{bond}}=932,62 \mathrm{eV}\right)$ on the surface spectra of the samples of elemental standards (Aurum, Argentum, Kuprum); calibration accuracy $\pm 0,03 \mathrm{eV}$. Charge samples were evaluated for Carbon C 1s spectra $(284,5 \mathrm{eV})$.

The analysis samples were prepared in a pre-fabricated thin layer of the sample ( $50,0 \mathrm{mg}$ ) in metallic In, which is located directly in the holder of the measuring chamber of the device.

For a detailed analysis of the chemical states of atoms we used the spectra decomposition into the individual components according to a program that takes into account the mixed Lorentz - Gaussian shape of the peaks and the area under the peaks while simultaneously optimizing the background parameters by using the minimizing principle of the number of bands needed to describe the experimental spectra.

The error in the peak position determining was $\pm 0,01 \mathrm{eV}$. The analysis of the chemical state of the atoms (Ferum, Oxygen and Carbon) on the surface of the samples consisted in a detailed study of the spectra of the electronic levels:Fe $2 p$ (705 - 740 $\mathrm{eV}), \mathrm{O} 1 \mathrm{~s}(525-554 \mathrm{eV})$ and $\mathrm{C} 1 \mathrm{~s}(275-295 \mathrm{eV})$ which allowed to quantify the phase composition of the surface of the samples.

\section{X-ray photoelectron spectroscopy (RPES or XPS)}

The chemical composition of the surface layers of the "lipid-NP $\mathrm{Fe}_{3} \mathrm{O}_{4}$ " system and the chemical state of the elements on its top arefurther investigated by the X-ray photoelectron spectroscopy (RPES) method. Fig. 1 shows the X-ray photoelectron spectra (RPES or XPS-spectra) of the internal electronic levels of $\mathrm{C} 1 \mathrm{~s}$ and Fe $2 p$ of the experimental samples 1 (Fig. 1, a, b) and 2 (Fig. 1, c, d) of the compositions "lipid$\mathrm{NP} \mathrm{Fe}_{3} \mathrm{O}_{4} "$. 

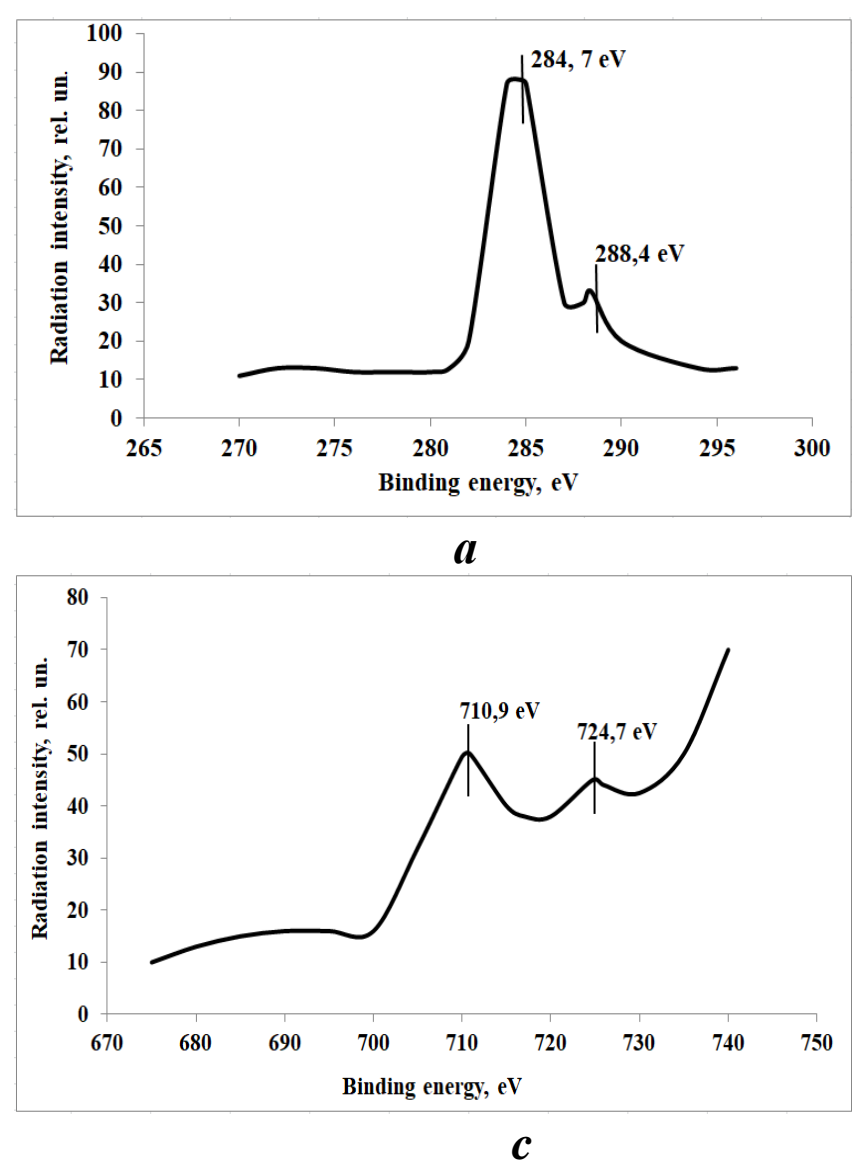

$c$

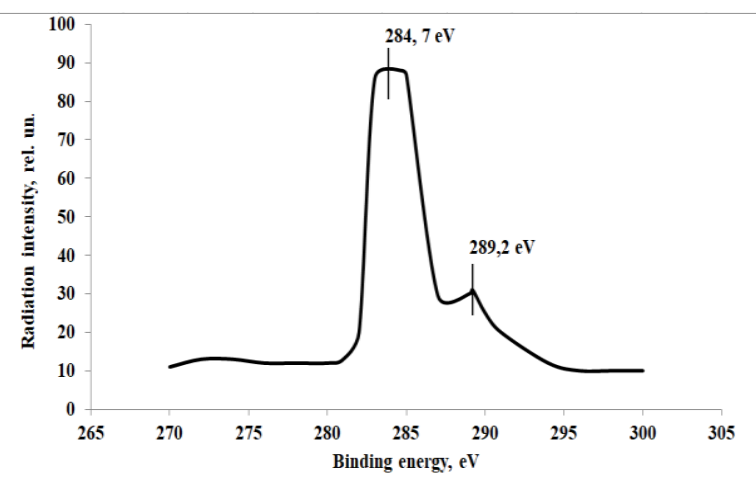

$\boldsymbol{b}$

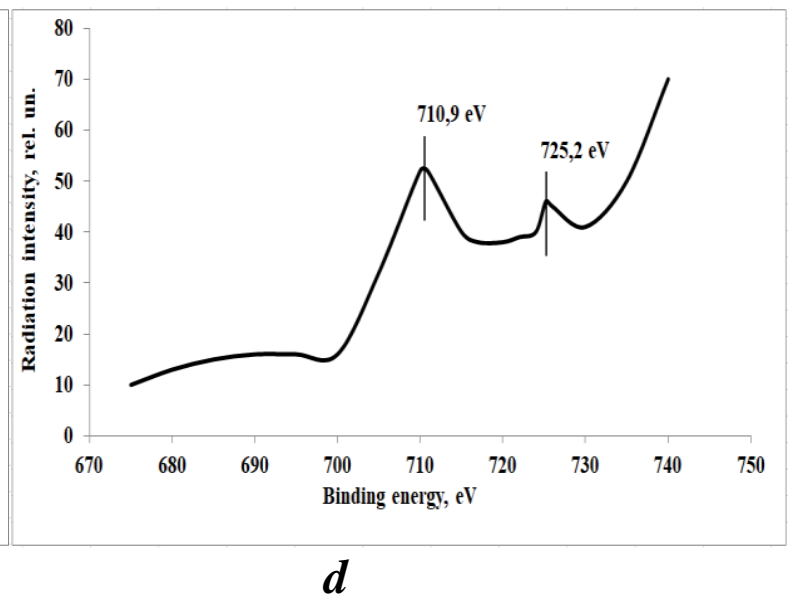

Fig. 1. XPS images of $\mathrm{C} 1 \mathrm{~s}$ and $\mathrm{Fe} 2 \mathrm{p}$ internal electronic levels: $\mathrm{C}$ 1s level of $\mathrm{Fe}_{3} \mathrm{O}_{4}$ nanoparticles coated with linoleic acid (a); $\mathrm{C} 1 \mathrm{~s}$ lvel of $\mathrm{Fe}_{3} \mathrm{O}_{4}$ nanoparticles coated with sunflower oil (b); $\mathrm{Fe} 2 \mathrm{p}$ level of $\mathrm{Fe}_{3} \mathrm{O}_{4}$ nanoparticles coated with linoleic acid (c) and $\mathrm{Fe}$ $2 \mathrm{p}$ level of $\mathrm{Fe}_{3} \mathrm{O}_{4}$ nanoparticles coated with sunflower oil (d)

\section{Results and discussion}

On the spectra (Fig. 1, a,b), there is no absorption band C $1 \mathrm{~s}$ at $290 \mathrm{eV}$ which corresponds to carboxylic Carbon $(-\mathrm{COOH})[20]$. And this indicates the absence of free carboxylic acid or ester group of triglyceride on $\mathrm{Fe}_{3} \mathrm{O}_{4}$ nanoparticles coated with lipids. Thepeak at 284,7 eV (Fig. 1, $a, b$ ) is attributed to the Carbon atoms in the aliphatic chain (C-C); and the peaks at 288,4 eV (Fig. 1, $a$ ) and 289,2 eV (Fig. 1, b) are related to carboxylate $\left(-\mathrm{COO}^{-}-\right)$. That agrees with the received data from the previous literature [9].

On the spectra (Fig. 1, $c, d$ ) the characteristic peak of oxides and ferric hydroxides at $710,9 \mathrm{eV}$ is not observed which characterizes the binding of energy to the electron of the basic level of $\mathrm{Fe} 2 \mathrm{p}_{3 / 2}$ [9]. However, in the experimental samples 1 (d) and 2 (e), 
an absorption band appeared in the area ofhigher binding energies $\quad 724,7 \mathrm{eV}$ (Fig. 1, в) and $725,2 \mathrm{eV}($ Fig. $1, d$ ). This absorption band is related to the carboxylate of the ferrum [9].

The spectra of X-ray photoelectron spectroscopy (RPEC) of the level C 1s and Fe $2 \mathrm{p}$ give one more confirmation of the chemical structure of the experimental samples of the system of "lipid-NPs $\mathrm{Fe}_{3} \mathrm{O}_{4}$ " system. Indicating the formation of the chemical bonds between the atoms of the Ferrums $\mathrm{NPs} \mathrm{Fe}_{3} \mathrm{O}_{4}$ and lipids Oxygen atoms (higher fatty acids, in particular, linoleic, and triglycerides of oils and fats, in particular sunflower oil).

\section{Conclusions}

The chemical composition of the surface layers in the experimental samples of the system "lipid-NPs $\mathrm{Fe}_{3} \mathrm{O}_{4}$ " was established by the method of X-ray photoelectron spectroscopy (RPES). On the spectra of samples 1, 2, the absorption band C 1s at 290 $\mathrm{eV}$ is not observed which corresponds to carboxylic Carbon $(-\mathrm{COOH})$. That is no free carbonic acid or ester group. The peak at $284,7 \mathrm{eV}$ (in samples 1,2) is attributed to Carbon atoms in the aliphatic chain $(\mathrm{C}-\mathrm{C}$ ); and the peaks at $288,4 \mathrm{eV}$ (sample 1) and $289,2 \mathrm{eV}$ (sample 2) are bound to carboxylate $\left(-\mathrm{COO}^{-}-\right.$). The spectra of samples 1,2 do not show the characteristic peak of oxides and ferrous hydroxides at 710,9 eV, however, the absorption band appeared at 724,7 eV (sample 1) and 725,2 eV (sample 2 ). This absorption band is related to the carboxylate of the ferrum. All this points to the formation of the chemical bonds between the atoms of Ferrum of $\mathrm{NPs}^{\mathrm{Fe}} \mathrm{O}_{3} \mathrm{O}_{4}$, and the lipids Oxygen atoms.

\section{References}

1. HerbacelA. PlusCitrusFibers - TypeN: Specification For Food Additives And Recipes, 2013. ( In English)

2. María J. Beriain, Inmaculada Gómez, Francisco C. Ibáñez, M. Victoria Sarriés, Ana I. Ordóñez. Chapter Food Quality: Balancing Health And Disease. A Volume In Handbook Of Food Bioengineering, 2018, 1, 74. ( In English)

3. Camila de Souza Paglarini, Guilherme de Figueiredo Furtado, João Paulo Biachi, Vitor Andre Silva Vidal, Marise Aparecida Rodrigues Pollonio:Journal of Food Engineering, 2018, 222, 29. ( In English)

4. TsykhanovskaI., AlexandrovA., Evlash V., Lazareva T., Bryzytska O.: Eastern european journal of advanced technology, 4, 94, 61. (In Ukrainian, doi: 10.15587/1729-4061.2018.140048.

5. Mahdavi M., Ahmad M., Haron M., Namvar F., Nadi B., Abrahman M.: Molecules, 2013, 18, 7533. ( In English), doi:10.3390/molecules18077533. 
Resource and Energy Saving Technologies of Production and Packing of Food Products as the Main Fundamentals of Their Competitiveness: Proceedings of the 7th International Specialized Scientific and Practical Conference, September 13, 2018. Kyiv, Ukraine

6. Alexandrov A., Tsykhanovska I., Evlash V., Svidlo K., Gontar T.: Eastern european journal of advanced technology, 5, 89, 61. (InUkrainian), doi: 10.15587/1729-4061.2017.111522.

7. Cihanovs'ka I., SkurihinaL., AleksandrovO., GontarT.: Progresivni tekhnikatatekhnologii harchovihvirobnictvrestorannogogospodarstva i torgivli: zb. nauk. pr. HDUHT, 2017, Harkiv, 1. (InUkrainian)

8. TsykhanovskaI., AlexandrovA., EvlashV., LazarevaT., Svidlo K., GontarT.: Eureka: LifeSciences, Estonia, Tallinn, 2017, 6, 43. ( In English), doi: 10.21303/2504-5695.2017.00511.

9. Tsykhanovska I., Alexandrov A., Evlash V., Lazareva T., Svidlo K., Gontar T.: Eureka: Life Sciences, Estonia, Tallinn, 2018, 2, 67. ( In English), doi: 10.21303/2504-5695.2018.00611. 